\title{
Older adults' perceptions of current and future hearing healthcare services in Australia, England, US and Canada
}

Catherine M McMahon', Cornetta L Mosley ${ }^{\mathrm{b}}$, M Kathleen PichoraFuller ${ }^{\mathrm{c}}$, Adrian C Davis ${ }^{\mathrm{d}}$, Carolyn R Baylore, Kathryn M Yorkston ${ }^{\mathrm{e}}$ and Kelly L Tremblay ${ }^{\ddagger}$

a HEAR Centre, Department of Linguistics, Macquarie University, Sydney, Australia

b Department of Speech Pathology and Audiology, University of South Alabama, US

c Department of Psychology, University of Toronto Mississauga, Canada

d The Ear Institute, University College London, UK

e Department of Rehabilitation Medicine, University of Washington, Seattle, US

${ }^{\dagger}$ Department of Speech and Hearing Sciences, University of Washington, Seattle, US

g Corresponding author: cath.mcmahon@mq.edu.au

\section{Article history}

Publication date: 2 December 2021

Citation: McMahon CM, Mosley CL, Pichora-Fuller MK, Davis AC, Baylor CR, Yorkston KM, Tremblay KL. Older adults' perceptions of current and future hearing healthcare services in Australia, England, US and Canada. Public Health Res Pract. 2021;31(5):e3152128. https://doi. org/10.17061/phrp3152128

\section{Key points}

- Recent and significant changes in policy and service delivery approaches, using over-the-counter models, have been developed to specifically address cost and accessibility of hearing healthcare ( $\mathrm{HHC})$ services

- This qualitative study demonstrates that, although cost remains a key challenge, long-standing issues of stigma and trust in the $\mathrm{HHC}$ system - originally identified about 30 years ago - remain despite significant technological advances and generational change

- Such barriers may remain an impediment to $\mathrm{HHC}$ uptake among older adults

\section{Abstract}

Objective: A high prevalence of hearing loss in older adults contrasts with a small proportion of people who seek help. Emerging developments in hearing healthcare $(\mathrm{HHC})$ could reduce costs but may not increase access. This study evaluated older adults' perceptions of current and future $\mathrm{HHC}$ services in Australia, England, US and Canada to explore potential levers and system improvements.

Methods: Semi-structured focus groups $(n=47)$ were conducted, and data were analysed using a directed content analysis. Participants were adults 60 years and older with a) no hearing problems; b) hearing problems and hearing aid use; and c) hearing problems and no hearing aid use.

Results: Perceived barriers, facilitators and preferences were largely consistent across countries, with stigma and trust in $\mathrm{HHC}$ being the barriers most often discussed.

Conclusion: Although cost and access were consistently deemed important, there may be limited change in help-seeking and $\mathrm{HHC}$ uptake unless the key barriers of trust and stigma are addressed. When seeking to undertake transformative change to healthcare it is important to engage recipients of care to understand existing barriers and coproduce a user-centered solution.

\section{Introduction}

Age-related hearing loss is a major public health problem that will increase with the ageing of the global population. Hearing loss in older adults is independently associated with higher risk of physical and cognitive decline, 
elevated healthcare costs, and increased mortality risks. ${ }^{1}$ Yet, despite age-related hearing loss being ranked as the fourth-leading contributor to years lived with a disability worldwide ${ }^{2}$, it remains poorly addressed and prioritised globally. Although evidence supports the benefits of hearing devices even for milder forms of hearing loss $^{3}$, device uptake and use in the ageing population is generally low. ${ }^{4}$ Cost is often cited as a major barrier to obtaining hearing aids but the prevalence of uptake is similar across many high-income countries, despite considerable differences in hearing health systems and out-of-pocket expenses. ${ }^{5}$

Hearing loss decreases workforce and social productivity. ${ }^{6}$ In this remarkable time of technological innovation, specialised amplification, signal processing and speech recognition technologies that may help people with hearing loss are converging with mainstream communication technologies. In parallel, health policies are changing to enable greater over-the-counter access. ${ }^{7}$ These trends have, in part, been supported by direct-toconsumer marketing in which technological innovations are marketed as a 'fix' for hearing loss, although such practices are condemned by consumer regulatory bodies. ${ }^{8}$ Of concern is that quality of care is hidden, bundled into the costs of devices and poorly recognised by consumers as a key element for achieving optimal hearing health outcomes. ${ }^{9}$ Greater availability of hearing technologies, coupled with demand for consumerdriven models of care, seem to be paving the way for massive disruption to the hearing healthcare $(\mathrm{HHC})$ industry. Redesign of care pathways and technologies requires engagement with current and future consumers to understand the existing challenges and to ensure that solutions are implemented with their needs and preferences in mind. Crucially, the notion of a quick fix does not recognise the long-term value of care for helping individuals age well as their communication needs change.

Using a qualitative approach, we sought opinions from older adults from four countries to identify the current factors affecting consumer uptake of $\mathrm{HHC}$ services and hearing aids, and consumer preferences for service improvement.

\section{Methods}

Forty-seven participants were recruited for four 2-hour focus groups ( $n=12$ per group, with one non-attendance) held in London, UK; Seattle, US; Sydney, Australia; and Toronto, Canada (see Table 1 for participant demographic information). Recruitment was conducted through TNS Global, a third-party London-based company with offices in the four countries. Participants were $\geq 60$ years of age and were recruited from one of three categories: no self-reported hearing problems; self-reported hearing problems but without hearing aid use; or self-reported hearing problems with hearing aid use. Recruitment was stratified in an attempt to include equal numbers of males and females in four age groups (60-64, 65-69, 70-74, and $\geq 75$ ) in each of the three hearing categories in each country. Most participants (70\%) were retired.

Semi-structured focus groups were conducted in person and facilitated by a locally based, professionally trained TNS-Global interviewer with no expert knowledge of hearing loss who used a single script developed with the lead research team (see Appendix 1, available from: doi.org/10.6084/m9.figshare.16893403). At least three members of the research team were present at each focus group. Real-time captioning (Communication Access Real-Time, CART) was used, and personal assistive listening solutions were available to all participants.

Table 1. Participant demographic information $(N=47)$

\begin{tabular}{|c|c|c|c|c|c|}
\hline & $\begin{array}{c}\text { US } \\
n(\%)\end{array}$ & $\begin{array}{c}\text { Australia } \\
n(\%)\end{array}$ & $\begin{array}{c}\text { Canada } \\
n(\%)\end{array}$ & $\begin{array}{c}\text { UK } \\
n(\%)\end{array}$ & $\begin{array}{c}\text { TOTAL } \\
n(\%)\end{array}$ \\
\hline \multicolumn{6}{|l|}{ Gender } \\
\hline Male & $6(50)$ & $6(50)$ & $7(64)$ & $6(50)$ & $25(53)$ \\
\hline Female & $6(50)$ & $6(50)$ & $4(36)$ & $6(50)$ & $22(47)$ \\
\hline \multicolumn{6}{|l|}{ Age } \\
\hline 60-64 years & $3(25)$ & $3(25)$ & $3(27)$ & $3(25)$ & $12(26)$ \\
\hline 65-69 years & $3(25)$ & $3(25)$ & $1(9)$ & $3(25)$ & $10(21)$ \\
\hline 70-74 years & $3(25)$ & $3(25)$ & $4(36)$ & $3(25)$ & $13(28)$ \\
\hline$\geq 75$ years & $3(25)$ & $3(25)$ & $3(27)$ & $3(25)$ & $12(26)$ \\
\hline \multicolumn{6}{|l|}{ Hearing status (self-reported) } \\
\hline Normal hearing & $4(33)$ & $2(17)$ & $4(36)$ & $4(33)$ & $14(30)$ \\
\hline Hearing problems with hearing aid use & $4(33)$ & $4(33)$ & $3(27)$ & $4(33)$ & $15(32)$ \\
\hline Hearing problems without hearing aid use & $4(33)$ & $6(50)$ & $4(36)$ & $4(33)$ & $18(38)$ \\
\hline
\end{tabular}


Audio recordings were transcribed verbatim by a TNS Global researcher and verified by a second person. Transcripts were uploaded into Dedoose (Los Angeles, CA: SocioCultural Research Consultants, LLC; 2016) and analysed using a directed content analysis approach. ${ }^{10}$ A coding dictionary was developed iteratively followed by comparison and consensus building by the qualitative research team to develop a final code dictionary. After coding and sorting the data, analysis continued to summarise and distil data into a parsimonious number of factors that reflected the most salient and common perspectives of participants, while noting diversity among experiences. Several methods were used to promote trustworthiness: recruitment was stratified, participation was fostered and facilitated, and the same script was used across focus groups. The research team contained diverse representation of clinical and research backgrounds. Common issues raised across all four focus groups, as well as the alignment of findings in this study with findings from previous research, suggest that saturation was approached in identifying key barriers and facilitators for seeking help for hearing problems.

\section{Ethics}

This study was approved by the University of Washington Health Internal Review Board - Human Subjects Division (\#47812); participants consented and were remunerated for their time.

\section{Results}

The results revealed four main factors that influenced participants' decisions to access HHC: 1) the experience of hearing loss and perceived need (according to self, significant others or authority figures); 2) stigma (self and societal); 3) HHC systems (healthcare professionals, pathways and authority figures); and 4) value of the service/technology (technological capabilities versus limitations and cost). Trust in HHC, stigma, and the cost and limitations of hearing technology, were the areas most often discussed. Although the narrative in each of the four countries was similar, the discussion was nuanced, with differences between countries evident when describing cost and trust in the context of sociopolitical-economic differences in healthcare systems (particularly for outof-pocket expenses). Participant quotes illustrating key points were chosen to represent the data.

\section{Barriers and facilitators}

\section{Perceived need}

Participants discussed levels of perceived need for help with hearing problems either as barriers to, or facilitators of, help-seeking. That is, having a lower perceived need served as a barrier to help-seeking, in part because the gradual onset of hearing loss made it difficult to perceive changes, or because the loss was not yet perceived as 'bad enough' to warrant seeing a professional. In contrast, perceiving adverse effects of hearing loss on everyday life was a facilitator of help-seeking, whether it was a relative difference between what the person and others could hear (e.g., television or radio); increased difficulty communicating at work or in noisy places; or frequently misunderstanding communication partners with strong accents. Another facilitator of help-seeking was the social withdrawal caused by the effort of listening and participating.

Many participants who had sought help described a breaking point when problems became serious enough for them to act. The breaking point was modulated by social influences such as the actions and reactions of communication partners. These included the perceived effects that hearing loss had on others during social interactions, creating 'awkward' or 'embarrassing' interactions, as well as the encouragement (or pressure) to seek help that came from family members, friends or people at work.

What motivates me is when I visit my son or he visits me and we're talking or I go with the grandchildren, we go to a concert or something at the school, he's continually telling me, 'Dad, you're talking too loud'. So, he's really the one that's continually on me saying I have to go and do something about it, but it's one of the things you put on the backburner. (Australia)

What pushed me into it was really, I got fed up trying to - saying to my kids, 'Sorry what did you say?' My wife saying, 'Would you please go and get some bloody hearing aids, we're not mumbling!' So that's what I really I guess got me going. (Canada)

\section{It was my daughter saying, 'Mum you're always} shouting,' and I didn't think I was. (UK)

I guess the breaking point was I was at church and I was standing next to somebody who was talking, and there was a whole group of us talking, but I didn't know what was spoken... I could hear the volume, but I couldn't tell what was said. (US)

\section{Stigma: hearing loss, hearing aids and negative views of ageing}

Stigma was one of the most pervasively reported barriers to seeking help for hearing problems. There were three inter-related sources of stigma: having hearing loss, wearing hearing aids, and ageing. Stigma was described as a key barrier to accepting and acknowledging hearing loss. Stigma was often associated with wearing hearing aids, regarded as a visible sign that would reveal one's disability and may be perceived as a sign of incompetence. The stigma attached to wearing hearing aids made some participants question their own readiness to seek help. 
Participants compared hearing loss to other agerelated health conditions, such as loss of vision or mobility, often concluding that hearing loss and hearing aids carried more stigma than other types of health aids (e.g., glasses). Notably, as well as concerns about discriminatory behaviours by others, self-stigma and negative views of ageing emerged as a barrier to seeking help. For some, acknowledging hearing problems entailed recognising and reconciling negative views of one's own ageing, which they associated with losing independence, becoming unable to carry out typical activities, losing social contacts and dying. In addition to hearing-related communication difficulties, participants believed their communication competence might be questioned based on ageist stereotypes. Beyond the issue of stigma, ageing was a self-perceived barrier to seeking help in other ways, for example, because of a diminished belief by themselves and others that older adults need to or can change.

If you admit that you're losing some of these faculties, that it's sort of the end is nigh or something ... and you're losing some of your independence. (Australia)

\section{I think there's a stigma still attached to admitting that you need a hearing aid. I think it's a sort of last frontier to admit that you're getting old. (Canada)}

I would say just one word that lots of people are reluctant to accept: that it's a vanity point. (UK)

I put off getting my hearing aid when I could have. I wasn't ready. I knew there was a problem but I was still working and I thought, 'Ooh, I'll put that thing on my ear, am I even thought of as capable?' (US)

\section{Hearing healthcare systems}

There was an evident lack of clarity about from whom to seek help, who to trust and how to seek help. The narratives across all four countries suggested that there was fragmentation of the care pathway, lack of an expert figure, and poor promotion of hearing screening, hearing aids and $\mathrm{HHC}$ by healthcare providers in general, thereby creating mistrust and barriers to referral. Participants raised the importance of having a physician's recommendation to see a $\mathrm{HHC}$ provider or obtain a hearing aid and wanted more (and trusted) information about $\mathrm{HHC}$ services and treatment options for hearing loss. Importantly, participants suggested that healthcare providers often normalised hearing loss as part of ageing, which discouraged help-seeking.

If the doctor said, 'If you don't get a hearing aid, you'll go down the tube quickly,' then you'll probably get a hearing aid. I think you need an authority figure to tell you. (Canada)

I don't trust private medicine whatsoever because you know the profit mark-up's there whereas when I go to the NHS [National Health Service] I know they're going to do what needs to be done medically, whereas when I'm going to private they're going to do what needs to be done to make a fast buck... (UK)

It would be nice if the Surgeon General would ... do a, you know, public service thing, and one thing that somebody kept telling me is when your hearing, you're losing your hearing, you should get your hearing aids soon... (US)

\section{Value (cost-benefit of technology)}

Participants overwhelmingly equated $\mathrm{HHC}$ service with purchasing hearing aids. They discussed the costs of hearing aids alone, but not the related costs of $\mathrm{HHC}$ services. Although some reported immediate benefits from hearing aids, many described their limitations, particularly in regard to filtering out background noise. Participants acknowledged that hearing aid technology had advanced but the limitations and perceived value of the technology to the individual were considered barriers to seeking help and continuing device use. Participants reported being motivated to seek help when told that managing hearing problems could help prevent further hearing loss or mitigate other health problems, such as dementia. Others, based on their own experience or that of others, felt that hearing aids would not deliver the benefits that they needed. Hearing loss was unfavourably compared with other health conditions, especially vision loss, where many participants believed that hearing aids did not offer as much benefit for hearing as glasses did for vision. Other barriers included physical discomfort and appearance - most participants preferred that their hearing aids not be visible to other people.

I have to tell you that when I first wore my hearing aid, I could not believe the difference. I thought I was hearing okay too, and the minute I put them on, I just - wow. (Australia)

I don't know where I'd be today without hearing aids - because I just feel so much better when I can hear people talking... (Canada)

I think a hearing aid, if it's going to give me a better quality of ... I don't want to be left out, I'm a social person ... and I want to be involved ... and you bet your life I would use it. (UK)

I've had hearing aids for a year and they've helped; they help a lot. They're not perfect, and I can't afford the fancy ones. (US)

\section{Future preferences}

The key themes for future preferences, aligned with the key barriers, included having an integrated and trusted $\mathrm{HHC}$ system, reducing the stigma of ageing and hearing 
loss, having better and more affordable technology, and having trustworthy services, providers and information (see Appendix 2 for supporting quotes from participants in the four countries, available from: doi.org/10.6084/ m9.figshare.16893418).

\section{Integrated and trusting hearing healthcare}

Participants believed that hearing problems were treated differently to other health conditions but strongly advocated for age-related hearing loss to be given the same consideration and ascribed the same importance. For example, they strongly recommended that hearing screenings be included in routine health examinations for older adults, with benefits such as earlier identification of hearing loss; reduced stress for individuals reporting hearing problems to their physicians; and reduced stigma associated with hearing problems. They believed that managing hearing problems alongside other health problems could increase awareness of its effects on health, safety and quality of life. This might prompt healthcare providers to address age-related hearing loss as a significant health issue in the broader context of promoting healthy ageing.

Participants recommended making information about hearing health and hearing services more trustworthy and accessible. Factors that could contribute to better quality care included receiving understandable information about the importance of addressing hearing problems early and effectively, and hearing aid return policies with full financial reimbursement if they were not satisfactory. There was diversity of opinion about whether to trust information about hearing problems presented in the media, advertisements, pamphlets in the mail, and unsolicited phone calls from hearing clinics. Some participants said having an easy rating system for hearing aids or hearing services could help people assess information. Participants also favoured having independent sources for advice without the pressure or commitment of having a hearing test or a sales pitch about hearing aids.

\section{Reduce stigma and increase public education}

Reducing the stigma of ageing and hearing problems was a high priority for participants across all four countries. Several strategies were suggested for increasing acceptance of hearing problems and hearing aids, such as making hearing aid use more appealing by including younger or attractive people in advertisements. Participants advocated for more public education and awareness programs, which might increase acceptance of people with hearing problems and promote hearing loss prevention through avoidance of loud noise exposure. Participants advocated for laws or policies that would reduce the noise in built environments and urban spaces to make them more accessible for people with hearing problems.

\section{Better technology}

Participants expressed a preference for technology that was more affordable and provided better quality sound. One common thread was a need to develop hearing aids that could overcome background noise. Participants spoke in favour of technologies that would help them blend in with other people instead of being readily identified as someone with hearing problems. Participants across focus groups even supported the idea of implantable hearing aids if they increased comfort, invisibility and convenience.

\section{Discussion}

The results of the current study demonstrate several factors that influence older adults' decisions about help-seeking for hearing problems, and their preferred changes to the HHC pathway. Specifically, participants described a breaking point for seeking help as a time when the disabling effects of hearing loss became apparent (i.e., when hearing loss became a significant barrier to important life experiences and social interactions). Reported barriers to HHC use included: significant societal stigma and self-stigma about hearing loss and ageing; mistrust of a fragmented system of care which lacked authority and transparent governance; and the high cost relative to low benefit evaluation of hearing technology. Future preferences were aligned with reported barriers. Preferences included: more education and awareness; policies and legislation to highlight the importance of hearing health; public awareness campaigns to reduce stigma; better integration of $\mathrm{HHC}$ with other types of healthcare; and more affordable hearing technologies with improved aesthetics and functionality in everyday life. We note that these opinions and frustrations of older adult consumers are consistent with those expressed in previous research in the past few decades; this suggests that previous efforts to improve $\mathrm{HHC}$ services and products are yet to address consumer concerns.

Systematic reviews have demonstrated that selfreported hearing disability is the strongest predictor of hearing aid use and satisfaction ${ }^{11}$, and that family members may provide motivation to seek help. ${ }^{12}$ Evidence suggests that hearing aids can benefit those with hearing $\operatorname{loss}^{3}$ and their significant others ${ }^{13}$, thus reducing the negative impacts arising from communication breakdowns. Humans are inherently social and preservation of communicative integrity can be fostered by adjusting the communication styles of the communication partner and/or the person with hearing loss, or by changing the communication environment, for example by talking in quieter spaces. ${ }^{14}$ Strained spousal relationships ${ }^{15}$ and/or social withdrawal ${ }^{16}$ are too often long-term consequences of unaddressed hearingrelated problems. Addressing hearing loss early can have positive benefits, including easier technological 
adoption, leading to greater adherence and use. ${ }^{17}$ However, seeking help is also influenced by cost-benefit evaluations, balancing social, emotional and financial costs with the perceived benefits or limitations of care outcomes (typically a hearing aid).

Stigma, trust in $\mathrm{HHC}$, cost and technological limitations were discussed as key barriers to seeking help, all of which have long been described in the literature. For example, in 1996 Hétu described how stigma, a primary reason given for workers not seeking help or wearing hearing aids, threatened social and personal identities. ${ }^{18}$ Further, a qualitative longitudinal study in 2010 demonstrated that stigma was related to changes in self-perception, ageism, and vanity (which is moderated by societal perceptions, norms and the significant other). ${ }^{19}$ The concept of a tipping or breaking point has also been described - influenced by the balance of coping and stress. ${ }^{20}$ Despite these concepts being first identified 25 years ago, public awareness campaigns and programs to address these $\mathrm{HHC}$ problems have been limited. ${ }^{21}$ Importantly, the past 25 years have seen rapid technological advances, generational shifts of those requiring hearing services from post-WWII generation to baby boomers, and greater information access through social media. Yet these challenges remain.

Interestingly, the cost and technological limitations of hearing aids were strongly foregrounded in the current study. However, there was little discussion about the value of care provided by HHC providers. ${ }^{22}$ Conspicuous by their absence, it seems that the healthcare system and its values have failed to adequately support and meet the needs of older adults with hearing problems, underscoring an urgent unaddressed need. Notwithstanding this, multiple studies have highlighted the importance of the therapeutic $\mathrm{HHC}$ relationship in providing supportive information, enhancing self-efficacy in using and incorporating hearing technologies into everyday life, and identifying when and what type of rehabilitative supports are needed. ${ }^{23}$ Addressing these factors may help to reduce the perception of stigma. $\mathrm{HHC}$ professionals have an important role in providing facilitatory elements of care, including selecting appropriate technologies for those with comorbidities (e.g., low vision), providing relevant supportive materials (e.g., large font manuals) and including family members in the rehabilitative process. ${ }^{24} \mathrm{~A}$ recent study suggests that adults fitted with hearing aids typically seek help beyond their $\mathrm{HHC}$ professional because of additional difficulties with their hearing devices. ${ }^{25}$ This offers opportunities for $\mathrm{HHC}$ professionals to be more engaged in resolving technological problems and providing socio-emotional support at acute high-need times along the care pathway. ${ }^{25}$ It also highlights potential benefits of partnerships between healthcare and not-for-profit organisations in co-managing chronic health conditions. It is important to define the role and value of $\mathrm{HHC}$ beyond fitting a hearing aid, especially in the face of industry disruption. A greater emphasis on the long-term value of engaging in a therapeutic relationship could increase the perceived value of $\mathrm{HHC}$ providers to those who require services that will need to be adapted as individuals age.

Despite concerns raised about hearing aids by participants in the current study, a recent Cochrane review demonstrated their effectiveness for older adults with milder forms of hearing loss ${ }^{3}$, suggesting that hearing technology can be beneficial across the span of hearing loss severity. However, the limitations of hearing aids in addressing the dominant complaint of adults with early stages of age-related hearing loss (hearing with background noise) have been recognised for decades. Current negative perceptions of hearing aids and low levels of trust in $\mathrm{HHC}$ providers have probably influenced the limited success of online hearing screening programs for older adults to seek help and manage their hearing health. ${ }^{26}$ Compliance in managing hearing health could be increased by embedding hearing screening within a routine health check; having trusted health professionals (physicians) motivating adults to seek help while addressing comorbid problems that may influence their communication needs and ability to use hearing technologies.

\section{Limitations}

Limitations of the study include its ability to fully reflect the country and system differences that might influence participants' experiences with and perspectives of differing $\mathrm{HHC}$ systems and hearing devices. To deepen our understanding of the barriers, facilitators and preferences expressed here, we have used these findings to inform the development of a four-country survey to gather a broader sample of consumer opinions in relation to their HHC experience.

\section{Conclusion}

Societal concerns of stigma, trust and value continue to be strongly related to the deeply embedded culture and structure of the $\mathrm{HHC}$ system. Policy changes are expected to expand the market and disrupt service delivery, and global advocacy opportunities will arise from the World Health Organization's recently released World Report on Hearing, which provides guidance on integrating ear and hearing care into national health plans. ${ }^{1}$ This provides an optimal time to rethink HHC. It will be important to align systems of care with population needs and preferences. Our findings suggest stigma, trust and value are necessary $\mathrm{HHC}$ ingredients that remain unaddressed. Therefore, when seeking to undertake transformative change to healthcare it is important to engage recipients of care to understand existing barriers and coproduce a user-centred solution that is valued and that would work across multiple stakeholders. ${ }^{27}$ This exploratory study highlights the universality of needs across these countries; 
collaborative, more efficient approaches are required to redesign and evaluate $\mathrm{HHC}$ systems for older adults.

\section{Acknowledgements}

This study was supported by a grant from the National Institutes of Health - NIDCD R21DC013161.

\section{Peer review and provenance}

Externally peer reviewed, invited. CMM is a Guest Editor of this themed issue of Public Health Research \& Practice. She had no part in the peer-review process for this paper.

\section{Competing interests}

$A D$ has received sponsorship from GN Resound to attend conferences, a fee from Natus for lecturing, and royalties paid to Manchester University (when he was employed there) for the intellectual property for Siemen's HearCheck Screener. KT has received fees from Lend an Ear Inc. for consultancy and developing educational presentations.

\section{Author contributions}

$\mathrm{KT}, \mathrm{AD}, \mathrm{CMM}$, and MP-F conceived and designed the program of research and attended focus groups. KT wrote the grant that funded this research as a former professor at the University of Washington (now retired). CLM, CB, and KY analysed the data; CMM, CLM, KT and MP-F wrote the paper; $C B, K Y$ and $A D$ contributed to and edited the manuscript.

\section{References}

1. World Health Organization. World report on hearing. Geneva: WHO; 2021 [cited 2021 Oct 7]. Available from: www.who.int/publications/i/item/world-report-on-hearing

2. Wilson BS, Tucci DL, Merson MH, O'Donoghue GM. Global hearing health care: new findings and perspectives. Lancet. 2017;390(10111):2503-15.

3. Ferguson MA, Kitterick PT, Chong LY, EdmondsonJones M, Barker F, Hoare DJ. Hearing aids for mild to moderate hearing loss in adults. Cochrane Database Syst Rev. 2017;9(9):CD012023.

4. Hartley D, Rochtchina E, Newall P, Golding M, Mitchell P. Use of hearing aids and assistive listening devices in an older Australian population. J Am Acad Audiol. 2010;21(10):642-53.

5. Moon IJ, Baek SY, Cho Y-S. Hearing aid use and associated factors in South Korea. Medicine (Baltimore). 2015;94(42):e1580.

6. Bainbridge KE, Wallhagen MI. Hearing loss in an aging American population: extent, impact, and management. Annu Rev Public Health. 2014;35:139-52.
7. Blustein J, Weinstein BE. Opening the market for lower cost hearing aids: regulatory change can improve the health of older Americans. Am J Public Health. 2016;106(6):1032-5.

8. Australian Competition \& Consumer Commission. ACCC takes action against hearing aid retailers for misleading pensioners. Canberra: ACCC; 2018 [cited 2021 Oct 7]. Available from: www.accc.gov.au/media-release/accctakes-action-against-hearing-aid-retailers-for-misleadingpensioners

9. Lin FR, Hazzard WR, Blazer DG. Priorities for improving hearing health care for adults: a report from the National Academies of Sciences, Engineering, and Medicine. JAMA. 2016;316(8):819-20.

10. Hsieh H-F, Shannon SE. Three approaches to qualitative content analysis. Quali Health Res. 2005;15(9):1277-88.

11. Knudsen LV, Öberg M, Nielsen C, Naylor G, Kramer SE. Factors influencing help seeking, hearing aid uptake, hearing aid use and satisfaction with hearing aids: a review of the literature. Trends Amplif. 2010;14(3):127-54.

12. Mahoney C, Stephens S, Cadge B. Who prompts patients to consult about hearing loss? Br J Audiol. 1996;30(3):153-8.

13. Stark P, Hickson L. Outcomes of hearing aid fitting for older people with hearing impairment and their significant others. Int J Audiol. 2004;43(7):390-8.

14. Hughes SE, Rapport FL, Boisvert I, McMahon CM, Hutchings HA. Patient-reported outcome measures (PROMs) for assessing perceived listening effort in hearing loss: protocol for a systematic review. BMJ Open. 2017;7(5):e014995.

15. Wallhagen MI, Strawbridge WJ, Shema SJ, Kaplan GA. Impact of self-assessed hearing loss on a spouse: a longitudinal analysis of couples. J Gerontology B Psychol SciSocSci. 2004;59(3):S190-S6.

16. Mick P, Pichora-Fuller MK. Is hearing loss associated with poorer health in older adults who might benefit from hearing screening? Ear Hear. 2016;37(3):e194-e201.

17. Davis A, Stephens D, Rayment A, Thomas K. Hearing impairments in middle age: the acceptability, benefit and cost of detection (ABCD). Br J Audiol. 1992;26(1):1-14.

18. Hétu R. The stigma attached to hearing impairment. Scand Audiol Suppl. 1996;25(43):12-24.

19. Wallhagen MI. The stigma of hearing loss. Gerontologist. 2010 Feb 1;50(1):66-75.

20. Southall K, Gagné JP, Jennings MB. Stigma: a negative and a positive influence on help-seeking for adults with acquired hearing loss. Int J Audiol. 2010;49(11):804-14.

21. Ruusuvuori JE, Aaltonen T, Koskela I, Ranta J, Lonka E, Salmenlinna I, Laakso M. Studies on stigma regarding hearing impairment and hearing aid use among adults of working age: a scoping review. Disab Rehab. 2021;43(3):436-46. 
22. Jennings MB. Audiologic rehabilitation needs of older adults with hearing loss: views on assistive technology uptake and appropriate support services Can J SpeechLanguage Pathol Audiol. 2005;29(3):112.

23. Hickson L, Meyer C, Lovelock K, Lampert M, Khan A. Factors associated with success with hearing aids in older adults. Int J Audiol. 2014;53(sup1):S18-S27.

24. Meyer C, Hickson L, Lovelock K, Lampert M, Khan A. An investigation of factors that influence help-seeking for hearing impairment in older adults. Int $\mathrm{J}$ Audiol. 2014;53(sup1):S3-S17.
25. Bennett RJ, Laplante-Lévesque A, Eikelboom RH. How do hearing aid owners respond to hearing aid problems? Ear Hear. 2019;40(1):77-87.

26. Meyer C, Hickson L, Khan A, Hartley D, Dillon H, Seymour J. Investigation of the actions taken by adults who failed a telephone-based hearing screen. Ear Hear. 2011;32(6):720-31.

27. Berwick DM. Improvement, trust, and the healthcare workforce. Qual Saf Health Care. 2003;12(6):448-52.

\section{Copyright: (c) (i) (2)}

(C) 2021 McMahon et al. This article is licensed under the Creative Commons Attribution-NonCommercial-ShareAlike 4.0 International Licence, which allows others to redistribute, adapt and share this work non-commercially provided they attribute the work and any adapted version of it is distributed under the same Creative Commons licence terms. See: www.creativecommons.org/licenses/by-nc-sa/4.0/ 